Advances in Dynamical Systems and Applications (ADSA).

ISSN 0973-5321 Volume 12, Number 1 (2017), pp. 15-20

(C) Research India Publications

https://dx.doi.org/10.37622/ADSA/12.1.2017.15-20

\title{
An elementary proof that symplectic matrices have determinant one
}

\author{
Donsub Rim \\ University of Washington, \\ Department of Applied Mathematics, Seattle, WA, 98195, USA.
}

\begin{abstract}
We give one more proof of the fact that symplectic matrices over real and complex fields have determinant one. While this has already been proved many times, there has been lasting interest in finding in an elementary proof $[2,5]$. The result is restricted to the real and complex case due to its reliance on field-dependent spectral theory, however in this setting we obtain a proof which is more elementary in the sense that it is direct and requires only well-known facts. Finally, an explicit formula for the determinant of conjugate symplectic matrices in terms of its square subblocks is given.
\end{abstract}

AMS subject classification: 15A15, 37J10.

Keywords: Symplectic Matrix, Determinants, Canonical transformations.

\section{Introduction}

A symplectic matrix is a matrix $A \in \mathbb{K}^{2 N \times 2 N}$ over a field $\mathbb{K}$ that is defined by the property

$$
A^{T} J A=J \quad \text { where } \quad J:=\left[\begin{array}{cc}
O & I_{N} \\
-I_{N} & O
\end{array}\right], \quad I_{N}:=\left(\text { identity in } \mathbb{K}^{N \times N} .\right)
$$

We are concerned with the problem of showing that $\operatorname{det}(A)=1$. In this paper we focus on the special case when $\mathbb{K}=\mathbb{R}$ or $\mathbb{C}$.

It is straightforward to show that such a matrix $A$ has $\operatorname{det}(A)= \pm 1$. However, there is an apparent lack of an entirely elementary proof verifying that $\operatorname{det}(A)$ is indeed equal to $+1[2,5]$, although various proofs have appeared in the past. Existing proofs require polar decomposition [3] or structured polar decomposition [6] in the least. Symplectic $\mathbb{G}$-reflectors developed in [7] can be exploited [5] to provide a proof. A proof utilizing 
the relationships of linearly independent rows and columns in the block structure of symplectic matrices, called the complementary bases theorem, appears in [2]. It is also widely known that a proof results from a study of Pfaffians [4]. We emphasize that some of these previous proofs, including the proof using the complementary bases theorem and that using Pfaffians, yield the result over arbitrary fields and are therefore more general.

The main contribution of this paper is to show that, when restricted to real matrices, there is a direct proof that requires only elementary linear algebra. With more work, this proof extends to complex matrices and this requires only basic topology of general linear groups. Moreover, for the conjugate symplectic matrices defined to satisfy $A^{*} J A=J$ rather than (1.1), the same approach yields an explicit formula (3.1) for the determinant in terms of its four square subblocks.

Although this is an independent result by the author, this precise approach for the real case has appeared previously in a reference written in Chinese by $\mathrm{Xu}$ [1], to our surprise. Perhaps not very widely known, this is the first appearance of the proof to the best of our knowledge.

\section{A proof that the determinant of a real or complex symplectic matrix is one}

Let us denote the set of $2 N \times 2 N$ symplectic matrices over field $\mathbb{K}$ by $\operatorname{Sp}(2 N, \mathbb{K})$.

A feature of the proofs below is that they follow a similar overall strategy for both cases, when $\mathbb{K}=\mathbb{R}$ and $\mathbb{K}=\mathbb{C}$. Nonetheless, in detail there is nontrivial difference arising from field-dependence, in the form of Lemma 2.2.

We first give the proof when $A$ is real.

Theorem 2.1. Let $A \in \operatorname{Sp}(2 N, \mathbb{R})$. Then $\operatorname{det}(A)=1$.

Proof. Taking the determinant on both sides of $A^{T} J A=J$,

$$
\operatorname{det}\left(A^{T} J A\right)=\operatorname{det}\left(A^{T}\right) \operatorname{det}(J) \operatorname{det}(A)=\operatorname{det}(J) .
$$

So we immediately have that $\operatorname{det}(A)= \pm 1$.

Then let us consider the matrix

$$
A^{T} A+I \text {. }
$$

Since $A^{T} A$ is symmetric positive definite, the eigenvalues of (2.2) are real and greater than 1 . Therefore its determinant, being the product of its eigenvalues, has $\operatorname{det}\left(A^{T} A+\right.$ I) $>1$.

Now as $\operatorname{det}(A) \neq 0, A$ is invertible. Using (1.1) we may write

$$
A^{T} A+I=A^{T}\left(A+A^{-T}\right)=A^{T}\left(A+J A J^{-1}\right) .
$$

Denote the four $N \times N$ subblocks of $A$ as follows,

$$
A=\left[\begin{array}{ll}
A_{11} & A_{12} \\
A_{21} & A_{22}
\end{array}\right], \quad \text { where } \quad A_{11}, A_{12}, A_{21}, A_{22} \in \mathbb{R}^{N \times N} .
$$


Then we compute

$$
\begin{aligned}
A+J A J^{-1} & =\left[\begin{array}{ll}
A_{11} & A_{12} \\
A_{21} & A_{22}
\end{array}\right]+\left[\begin{array}{cc}
O & I_{N} \\
-I_{N} & O
\end{array}\right]\left[\begin{array}{ll}
A_{11} & A_{12} \\
A_{21} & A_{22}
\end{array}\right]\left[\begin{array}{cc}
O & -I_{N} \\
I_{N} & O
\end{array}\right] \\
& =\left[\begin{array}{ll}
A_{11} & A_{12} \\
A_{21} & A_{22}
\end{array}\right]+\left[\begin{array}{cc}
A_{22} & -A_{21} \\
-A_{12} & A_{11}
\end{array}\right]=\left[\begin{array}{cc}
A_{11}+A_{22} & A_{12}-A_{21} \\
-A_{12}+A_{21} & A_{11}+A_{22}
\end{array}\right] .
\end{aligned}
$$

Writing the blocks as $C:=A_{11}+A_{22}$ and $D:=A_{12}-A_{21}$, we make use of a unitary transform

$$
\begin{aligned}
A+J A J^{-1} & =\left[\begin{array}{cc}
C & D \\
-D & C
\end{array}\right] \\
& =\frac{1}{\sqrt{2}}\left[\begin{array}{cc}
I_{N} & I_{N} \\
i I_{N} & -i I_{N}
\end{array}\right]\left[\begin{array}{cc}
C+i D & O \\
O & C-i D
\end{array}\right] \frac{1}{\sqrt{2}}\left[\begin{array}{cc}
I_{N} & -i I_{N} \\
I_{N} & i I_{N}
\end{array}\right] .
\end{aligned}
$$

We plug this factorization into (2.3). Note that $C, D$ are both real. This allows the complex conjugation to commute with the determinant (as it is a polynomial of its entries)

$$
\begin{aligned}
0<1<\operatorname{det}\left(A^{T} A+I\right) & =\operatorname{det}\left(A^{T}\left(A+J A J^{-1}\right)\right) \\
& =\operatorname{det}(A) \operatorname{det}(C+i D) \operatorname{det}(C-i D) \\
& =\operatorname{det}(A) \operatorname{det}(C+i D) \operatorname{det}(\overline{C+i D}) \\
& =\operatorname{det}(A) \operatorname{det}(C+i D) \overline{\operatorname{det}(C+i D)}=\operatorname{det}(A)|\operatorname{det}(C+i D)|^{2} .
\end{aligned}
$$

Clearly, none of the two determinants on the RHS can be zero, so we may conclude $|\operatorname{det}(C+i D)|^{2}>0$. Dividing this through on both sides, we have $\operatorname{det}(A)>0$, and thus $\operatorname{det}(A)=1$.

The unitary transform (2.7) appears in the final part of a proof that the determinant of any real symplectic matrix is +1 using polar decomposition in [3] although there it is not used in conjunction with (2.2). In our proof its use was in showing that

$$
\operatorname{det}\left(\left[\begin{array}{cc}
C & D \\
-D & C
\end{array}\right]\right) \geq 0 \quad \text { for any } C, D \in \mathbb{R}^{N \times N}
$$

The next lemma is a generalization of (2.8) to complex matrices, and is the main hurdle in applying the above approach to the complex field. It arises naturally in the treatment of quaternionic matrices, and was proved in $[8,9]$. We simply reproduce the latter proof here for completeness.

Lemma 2.2. Let $C, D \in \mathbb{C}^{N \times N}$. Then $\operatorname{det}\left(\left[\begin{array}{cc}C & D \\ -\bar{D} & \bar{C}\end{array}\right]\right) \geq 0$.

Proof. Let us first assume that $C$ is invertible. 
Then letting $E:=C^{-1} D, \operatorname{det}(\bar{E} E+I) \geq 0$ since eigenvalues of $\bar{E} E$ occur in conjugate pairs and every negative eigenvalue of $\bar{E} E$ has even algebraic multiplicity [10]. Note that $E$ and $I$ commute, which implies that

$$
\operatorname{det}\left(\left[\begin{array}{cc}
I & E \\
-\bar{E} & I
\end{array}\right]\right) \geq 0
$$

Then we have,

$$
\left[\begin{array}{cc}
C^{-1} & 0 \\
0 & (\bar{C})^{-1}
\end{array}\right]\left[\begin{array}{cc}
C & D \\
-\bar{D} & \bar{C}
\end{array}\right]=\left[\begin{array}{cc}
I & C^{-1} D \\
-C^{-1} D & I
\end{array}\right]=\left[\begin{array}{cc}
I & E \\
-\bar{E} & I
\end{array}\right] .
$$

Taking the determinant on both sides, we have the result. The Lemma follows by continuity.

Now we prove the complex case. Given Lemma 2.2 the proof is very similar to the real case.

Theorem 2.3. Let $A \in \operatorname{Sp}(2 N, \mathbb{C})$. Then $\operatorname{det}(A)=1$.

Proof. If $A$ is symplectic then we again have from (2.1) that $\operatorname{det}(A)= \pm 1$. Consider the matrix

$$
A^{*} A+I \text {. }
$$

The matrix $A^{*} A$ is Hermitian positive definite and therefore the matrix (2.9) has eigenvalues greater than one, and thus $\operatorname{det}\left(A^{*} A+I\right)>1$. Then we factor,

$$
A^{*} A+I=A^{*}\left(A+A^{-*}\right)=A^{*}\left(A+\overline{A^{-T}}\right) .
$$

Then we again use the definition (1.1) and with the notation (2.4) for subblocks,

$$
\begin{aligned}
A+\overline{J A J^{-1}} & =\left[\begin{array}{ll}
A_{11} & A_{12} \\
A_{21} & A_{22}
\end{array}\right]+\overline{\left[\begin{array}{cc}
A_{22} & -A_{21} \\
-A_{12} & A_{11}
\end{array}\right]} \\
& =\left[\begin{array}{rr}
A_{11}+\overline{A_{22}} & \frac{A_{12}-\overline{A_{21}}}{-\overline{A_{12}}+A_{21}} \\
\overline{A_{11}}+A_{22}
\end{array}\right]=\left[\begin{array}{cc}
C & D \\
-\bar{D} & \bar{C}
\end{array}\right],
\end{aligned}
$$

if we let $C:=A_{11}+\overline{A_{22}}$ and $D:=A_{12}-\overline{A_{21}}$. Therefore we have from (2.10)

$0<1<\operatorname{det}\left(A^{*}\left(A+A^{-*}\right)\right)=\overline{\operatorname{det}(A)} \operatorname{det}\left(\left[\begin{array}{cc}C & D \\ -\bar{D} & \bar{C}\end{array}\right]\right)=\operatorname{det}(A) \operatorname{det}\left(\left[\begin{array}{cc}C & D \\ -\bar{D} & \bar{C}\end{array}\right]\right)$.

By Lemma 2.2 we necessarily have that $\operatorname{det}\left(\left[\begin{array}{cc}C & D \\ -\bar{D} & \bar{C}\end{array}\right]\right)>0$, so we divide and conclude $\operatorname{det}(A)>0$, showing that $\operatorname{det}(A)=1$. 


\section{An explicit formula for determinant of conjugate symplectic matrices}

Conjugate symplectic matrices $A \in \mathbb{C}^{2 N \times 2 N}$ are defined similarly to symplectic matrices, by replacing the transpose in (1.1) with the conjugate transpose,

$$
A^{*} J A=J .
$$

Again, it immediately follows that $|\operatorname{det}(A)|=1$, however $\operatorname{det}(A)$ may take on any complex value on the unit circle [5]. Turning our attention to (2.9) once more,

$$
A^{*} A+I=A^{*}\left(A+A^{-*}\right)=A^{*}\left(A+J A J^{-1}\right) .
$$

With the previous notation for the $N \times N$ subblocks of $A$ and with $C, D$ defined as

$$
A=\left[\begin{array}{ll}
A_{11} & A_{12} \\
A_{21} & A_{22}
\end{array}\right], \quad C:=A_{11}+A_{22}, \quad D:=A_{12}-A_{21},
$$

we obtain (2.7) but for a complex matrix. Then it follows that

$$
\begin{aligned}
1<\overline{\operatorname{det}(A)} \operatorname{det}\left(\left[\begin{array}{rr}
C & D \\
-D & C
\end{array}\right]\right) & =\overline{\operatorname{det}(A)} \operatorname{det}(C+i D) \operatorname{det}(C-i D) \\
& =\operatorname{det}(A) \overline{\operatorname{det}\left(C^{2}+D^{2}-i[C, D]\right)},
\end{aligned}
$$

where $[C, D]:=C D-D C$ is the commutator.

This determines the phase and therefore $\operatorname{det}(A)$, yielding the formula

$$
\operatorname{det}(A)=\frac{\operatorname{det}\left(\left(A_{11}+A_{22}\right)^{2}+\left(A_{12}-A_{21}\right)^{2}-i\left[A_{11}+A_{22}, A_{12}-A_{21}\right]\right)}{\left|\operatorname{det}\left(\left(A_{11}+A_{22}\right)^{2}+\left(A_{12}-A_{21}\right)^{2}-i\left[A_{11}+A_{22}, A_{12}-A_{21}\right]\right)\right|} .
$$

\section{Acknowledgements}

The author would like to express thanks to Prof. Froilán M. Dopico and Prof. Ioana Dumitriu for carefully reading this paper and providing valuable comments, and thanks Prof. Bernard Deconinck for introducing him to this problem.

He also thanks Dr. Meiyue Shao for bringing to attention the reference [1].

\section{References}

[1] Y. Xu, Linear Algebra and Matrix Theory, (Chinese) 2nd Edition, Higher Education Press, Beijing, China, 2008.

[2] F. M. Dopico, C. R. Johnson, Complementary bases in symplectic matrices and a proof that their determinant is one, Linear Algebra Appl. 419 (2006), 772-778. 
[3] K.L. Meyer, G.R. Hall. Introduction to Hamiltonian Dynamical Systems and the N-Body Problem, Volume 90 of Applied Mathematical Sciences, Springer, New York., 1991.

[4] E. Artin. Geometric Algebra, Interscience Tracts in Pure and Applied Mathematics. Interscience Publishers Inc., New York, 1957.

[5] D.S. Mackey, N. Mackey, On the determinant of symplectic matrices, Numerical Analysis Report No. 422, Manchester Centre for Computational Mathematics, Manchester, England, (2003).

[6] D.S. Mackey, N. Mackey, F. Tisseur, Structured tools for structured matrices, Electron. J. Linear Algebra 419 (2003), 106-145.

[7] D.S. Mackey, N. Mackey, F. Tisseur, $\mathbb{G}$-reflectors in scalar product spaces. Linear Algebra Appl. 385 (2004), 187-213.

[8] H. Aslaksen, Quaternionic Determinants, Math. Intell. 18(3) (1996), 57-65.

[9] F. Zhang, Quaternions and matrices of quaternions, Linear Algebra Appl. 251 (1997), 21-57.

[10] R. A. Horn and C.R. Johnson, Matrix Analysis, Cambridge University Press, 1990. 